\title{
Colpopexia sacrospinosa: una alternativa razonable para el manejo del prolapso de cúpula vaginal por vía vaginal
}

\author{
Bayron Manuel Ruiz*; Pedro Alfonso Cortázar**; José Fernando Celades***
}

\begin{abstract}
RESUMEN: La vía vaginal para la corrección del prolapso de cúpula vaginal incluyendo cisto recto y enterocele, ha demostrado ser una opción razonable con bajo porcentaje de complicaciones y resultados satisfactorios. Se describe la técnica de fijación de la cúpula vaginal al ligamento sacrospinoso por vía vaginal, comentando sus ventajas y sus desventajas. Se presenta un grupo de 15 pacientes sometidas a esta técnica quirúrgica durante un lapso de tres años con resultados exitosos en 12 de ellas, con una sola complicación infecciosa y estancia hospitalaria promedio 72 horas. Se plantea la posibilidad de estudios comparativos con las técnicas por vía abdominal.
\end{abstract}

PALABRAS CLAVES: Colpopexia, prolapso de cúpula vaginal, fijación sacrospinoso.

SUMMARY: Vaginal way for correction of vaginal stump prolapse including cysto recto and enterocele, has been demonstrated to be a worthy chance, with low rate of complications and satisfactory results. The surgycal technique for sacrospinous fixation of the vaginal vault by the vaginal approach is described, talking about is advantages and disadvantages. A group of 15 patients who underwent this surgical tecnic during a 3 years period whith succesfull results in 12 of them is shown, with just an infection complication and hospital discharge at 72 hours. The posibility of comparative trails with abdominal way techniques is suggested.

KEY WORDS: Colpopexy, vaginal stump prolapse, sacrospinous fixation.

\section{Introducción}

A través de los años la corrección quirúrgica del Prolapso de Cúpula Vaginal ha sido un reto para los Ginecólogos. Innumerables técnicas han sido descritas, cada una de las cuales en opinión de sus defensores, es la más adecuada; pero finalmente hay dos grandes vías de acceso (Abdominal y Vaginal) para obtener los principales objetivos que son (1):

1. Mejorar la sintomatología

2. Reponer la anatomía normal

3. Preservar en lo posible la función sexual

En otras publicaciones (1-9) se destacan las ventajas y experiencias con las técnicas que emplean la vía abdominal.

La fijación de la cúpula vaginal a uno o ambos ligamentos Sacrociáticos (Colpopexia Sacrospinosa) por vía vaginal, ha logrado un alto porcentaje de éxitos en las metas propuestas ya sea como técnica primaria cuando es evidente la ausencia de tejidos apropiados a los cuales conectar el muñón vaginal en la Histerectomía Vaginal (10), o como técnica secundaria en el prolapso de cúpula vaginal después de Histerectomía, permitiendo al mismo tiempo y a través de la misma exposición operatoria la corrección de enteroceles, descensos de vejiga y recto, reconstrucción del bloque muscular perineal y evitando el ingreso a la cavidad abdominal, siendo todos los ante-

\footnotetext{
* Ginecobstetra. Profesor Auxiliar. Universidad del Quindío. Coordinador Servicio de Ginecobstetricia. ISS. Seccional Quindío. ** Ginecobstetra. ISS. Seccional Quindío.

*** Ginecobstetra. Hospital San Juan de Dios. Armenia
}

riores, elementos deseables para un menor trauma quirúrgico y una mejor evolución postoperatoria (10-12).

Cuando la inversión de la cúpula vaginal se acompaña de cisto y rectocele, la adición de la fijación de la cúpula al ligamento sacrociático prolonga 15 a 20 minutos la corrección convencional de celes, con evidentes ventajas sobre la corrección abdominal del prolapso de cúpula en el mismo acto quirúrgico (10).

En la presente revisión se analiza la técnica quirúrgi$\mathrm{ca}$, sus posibles ventajas y desventajas y se presenta un grupo de pacientes manejadas por los autores.

\section{Revisión histórica}

En 1892 Zweifel (13), describió la Fijación Sacrotuberosa de la Cúpula Vaginal, Miller (14) en 1927 describió un método transvaginal para corregir la eversión de la vagina usando el ligamento uterosacro, también así lo hizo Mengerts (15) en 1936.

Amreich (16) publicó su Fijación Sacrotuberosa inicialmente por vía coccígea y más tarde (1951) por vía vaginal, siendo bautizados por Sederl en 1958 Amreich I y II respectivamente; Mac Call (17) utilizó los ligamentos uterosacros aproximándolos y suturando la vagina debajo del "puente" formado por ellos.

En 1957 Richter (18) reintrodujo en Europa el concepto de Fijación Sacrotuberosa y desde 1968 (19-20) describió el uso del Ligamento Sacrospinoso como un avance de la técnica.

En 1971 Randall y Nichols (21) publicaron su serie inicial de 18 (dieciocho) pacientes manejadas por vía vaginal con fijación de la cúpula al ligamento sacrocospinoso, popularizando la técnica en E.E.U.U, 
continuando en años posteriores (10) con series mayores y resultados exitosos.

En 1988 Morley y Delancey (22) publican su serie de 100 (cien) pacientes tratadas con Colpopexia Sacrospinosa en la cual reafirman el éxito de la cirugía, puntualizando en el uso del material absorbible y las ventajas de la fijación a un solo ligamento sacrospinoso.

A nivel nacional se inició en 1986 la práctica de esta técnica quirúrgica por parte de los doctores Oscar Gómez Ceballos y Orlando Osorio en la ciudad de Manizales, siendo incorporada al programa de Residencia de Ginecobstetricia en la Universidad de Caldas y de allí a otras universidades del país.

Recientemente Peters y Christenson (23) 1995, publicaron su experiencia comparando la técnica descrita con la fijación del ápex vaginal a la fascia del músculo coccígeo obteniendo resultados similares.

\section{Materiales y métodos}

En el período mayo de 199 a abril de 1994 se practicaron 20 fijaciones de cúpula al ligamento sacrospinoso por vía vaginal en 19 pacientes (una fue reintervenida con éxito después de falla del procedimiento inicial) en la Clínica San José del I.S.S. Seccional Quindío en Armenia.

Se incluyeron en el estudio sólo 15 pacientes con edad promedio 58,8 años (rango 39 a 73 años, 12 de ellas en el grupo de edad de 45 a 65 años), a quienes se pudo realizar controles periódicos hasta mayo de 1995, las cuatro restantes se extraviaron después del primer control.

11 pacientes tenían antecedente de histerectomía abdominal, 3 histerectomía vaginal y una fue sometida a Fijación Profiláctica de la Cúpula durante histerectomía vaginal por extrema laxitud de los tejidos (prolapso integral).

Las intervenciones fueron realizadas por los autores quienes realizaron el seguimiento post-operatorio inicialmente trimestral por seis meses y después semestral.

El período mínimo postquirúrgico para inclusión en el estudio fue de un año, evaluando los resultados por interrogatorio y examen físico.

Se consideró recidiva la presencia de cualquier grado de prolapso de cúpula aún a pesar de manifestaciones subjetivas de bienestar por parte de la paciente.

\section{Descripción de la técnica quirúrgica}

La técnica descrita a continuación es la empleada por los autores basados en la formación inicial en el programa de Residencia y en la experiencia adquirida con un número importante de pacientes. Para un conocimiento detallado de la técnica nos remitimos a las excelentes descripciones gráficas de Morley (22) y Richter (24).

Con la paciente en posición de litotomía dorsal, previa asepsia y antisepsia bajo anestesia general o regional, se evacúa vejiga, se identifica cúpula vaginal practicando incisión en elipse transversal que incluye la zona cicatrizal* ${ }^{*}$, se diseca espacio prevesical rechazando vejiga separándola de la cara anterior de la vagina, exponiendo saco del enterocele (si existe) el cual se diseca y liga a nivel alto con material inabsorbible. Se corrige cistocele con técnica usual.
Se realiza incisión en "V" en periné con disección y corte longitudinal de la cara posterior de vagina hasta el nivel de la cúpula (* En algunos casos se inicia la cirugía por esta vía). Separando piel y pared vaginal exponiendo espacio rectovaginal avascular; se rechaza medialmente recto exponiendo en forma bilateral los músculos elevadores del ano y se diseca digitalmente el espacio pararrectal hasta la espina ciática (Generalmente la derecha en los cirujanos diestros ) para lo cual se practica apertura digital del "Pilar Rectal" (Ligamento Rectovaginal), identificando el ligamento sacrociático cubierto por las fibras del músculo coccígeo. Se colocan tres separadores medianos maleables rechazando: hacia arriba los ligamentos cardinales y el uréter, medialmente el recto, y hacia afuera la pared pélvica, exponiendo así el área correspondiente al ligamento el cual semeja una "hamaca".

Queda resuelta toda confusión relativa a la identificación del ligamento sacrociático menor cuando el cirujano palpa la estructura firme y resistente del ligamento en la profundidad del músculo isquiococcígeo el cual discurre del sacro a la espina ciática. No es necesario disecar exhaustivamente el músculo del ligamento ya que el trauma puede ocasionar un sangrado molesto e indeseable.

Con pinza de Allys se toma el ligamento comprobando su firmeza al traccionar y percibir que se mueve todo el cuerpo de la paciente. Se coloca un punto de material absorbible calibre 1 generalmente Poliglactina (Vicryl) un centímetro medial de la espina ciática a través del ligamento (evitando rodearlo por el peligro de lesión vascular o de nervio pudendo). Se inserta un segundo punto un centímetro medial al primero. La tracción de cada uno de los hilos de sutura comprueba la buena toma del ligamento, luego ambas suturas se colocan en los bordes de la cúpula y se reparan.

Verificada la hemostasia se aproximan los músculos elevadores con material inabsorbible calibre 1 sin anudar. Se reseca la pared vaginal excedente y se aproxima ésta con puntos separados de catgut cromado calibre cero o dos ceros, iniciando en la parte más distal a nivel de la cúpula hasta afrontar el tercio superior o medio del trayecto vaginal y sólo entonces se anudan y cortan sucesivamente los puntos de fijación verificando que exista firme oposición de los tejidos entre la superficie del ligamento y el borde la cúpula. Se anudan los puntos de aproximación de los elevadores y se termina la colpoperineorrafía en forma usual. En todas las pacientes se deja sonda vesical o cistofló por 24 ó 48 horas y mecha vaginal compresiva por 12 a 24 horas.

\section{Comentarios}

En el manejo del prolapso de cúpula vaginal por vía vaginal se plantean en la actualidad tres opciones principales (24):

Los pesarios

La Colpocleisis

La fijación al ligamento sacrospinoso

Teniendo en cuenta que en el grupo de edad más afectado -45 a 65 años- (7) la vida sexual activa es un importante factor, los pesarios y la colpocleisis no brindan un enfoque satisfactorio a la corrección del problema, sobre todo por la alta incidencia de incontinencia 
urinaria con ésta última, quedando relegada a pacientes mayores de 80 años (23) generalmente con cirugías previas no exitosas. Los pesarios podrían ser una medida temporal en pacientes candidatas a cirugía.

La Colpopexia Sacrospinosa vaginal retorna la cúpula al plano horizontal sobre el plato elevador y logra en la mayoría de las veces una razonable vida sexual $(12,22,25$ 27), permitiendo la restauración del canal vaginal a un estado funcional, con un eje superior normal inclinado horizontalmente y fijo por encima de la placa de los elevadores con lo cual disminuyen las posibilidades de recurrencia; así mismo el restablecimiento de la profundidad vaginal por reducción de los pliegues en acordeón de la vagina invertida elevará la porción del ligamento pubouretral posterior del diafragma urogenital a un nivel más alto en el interior de la cavidad pélvica mejorando las relaciones retropúbicas de los órganos genitourinarios (27).

En pacientes con prolapso genital total (integral) es recomendable la fijación "profiláctica" de la cúpula al ligamento sacrociático en vista de la gran probabilidad de que la debilidad de los elementos de sostén, evidente por el prolapso masivo, sea la base de un prolapso de cúpula posteriormente $(21-22,24)$.

Teniendo en cuenta la alta frecuencia de cisto, recto y enterocele acompañando el prolapso de cúpula (24), es relevante la ventaja de la vía única para la corrección de todos estos defectos anatómicos y funcionales en lo referente a tiempo quirúrgico, estancia hospitalaria y evolución post operatoria.

Las principales complicaciones de la cirugía $(10,22$, 24) son:

Recidiva del prolapso, incontinencia o retención urinaria, sangrado excesivo, morbilidad febril, atrapamiento del nervio ciático, acortamiento vaginal excesivo, lesión del recto.

Otras complicaciones de rara observación: evisceración masiva post quirúrgica (28) o muerte de la paciente (10), han sido descritas.

La técnica quirúrgica puede tener algunas variantes dignas de comentar:

Inicialmente se describió la fijación bilateral a los ligamentos sacrospinosos (22) pero se ha demostrado que la fijación a un sólo ligamento es igualmente exitosa considerando que la desviación de la cúpula lateralmente puede permitir que las fuerzas ejercidas por las vísceras abdominales en la bipedestación contribuyan a presionarla contra el diafragma pélvico (25).

El uso de material resorbible obedece a la posibilidad de compromiso inadvertido de nervios pudendo o ciático con uno de los puntos de fijación (dada su cercanía a la zona de transficción) lo cual origina molestias dolorosas que ceden paulatinamente con la reabsorción del material de sutura sin detrimento de la función de sostén, la cual dependería de la adhesión y cicatrización por íntimo contacto entre la cúpula y la superficie del ligamento; sin embargo algunos autores publican resultados satisfactorios con material inabsorbible $(22,25-26)$.

Recientemente se ha descrito la fijación del ápex vaginal a la fascia del coccígeo en el manejo quirúrgico del prolapso de cúpula vaginal por histerectomía (23), obteniendo similares resultados con una tasa de curación proyectada a dos años del $96 \%$, superior a la de las pacientes sometidas a fijación de cúpula al ligamento sacrospinoso ( $80 \%$ en el mismo estudio).

\section{Resultados}

En la Tabla 1 se resumen los resultados obtenidos con el grupo de pacientes estudiadas:

\section{Recidiva:}

En cuatro pacientes se presentó reaparición del prolapso, una de ellas fue reintervenida exitosamente con fijación de la cúpula al ligamento sacrospinoso contralateral.

Dos de estas pacientes son diabéticas y tres tenían corrección fallida previa del prolapso de cúpula por vía abdominal.

\section{Complicaciones:}

Exceptuando una paciente con infección en cúpula, la cual ameritó manejo antibiótico y hospitalización de ocho días, no hubo complicaciones intra ni post operatorias.

La paciente en mención evolucionó satisfactoriamente y actualmente se encuentra asintomática con cúpula sostenida.

\section{Dolor:}

Una paciente manifestó dolor persistente post colpopexia, siendo el único caso que presentó dehiscencia inmediata de la sutura de fijación; reintervenida dos años más tarde con excelente evolución, cúpula sostenida, sin dolor durante la evaluación.

\section{Retención urinaria:}

No hubo casos de retención urinaria en el post quirúrgico inmediato, ni en evaluaciones posteriores.

\section{Cistocele:}

De las 12 pacientes con cúpula sostenida en la última evaluación 10 presentaron cistocele, siendo grado III en cuatro de ellas, grado II en las seis restantes.

\section{Incontinencia urinaria:}

Cuatro pacientes tenían leve incontinencia preoperatoria, mejorando totalmente tres de ellas con la cirugía, persistiendo una con incontinencia leve.

Una de las 11 pacientes que no tenían incontinencia previa a la cirugía se quejó de "Incomodidad" para la micción sin verdadera incontinencia.

\section{Vida sexual:}

Cinco pacientes con edades entre 63 y 73 años no tenían vida sexual activa prequirúrgica y no la reiniciaron después.

Diez pacientes entre 39 y 61 años tenían vida sexual activa, siendo satisfactoria en seis de ellas. De estas últimas, cuatro continuaron vida sexual satisfactoria post colpopexia, dos no la reiniciaron (por temor a complicaciones post quirúrgicas). Dos de las cuatro que inicialmente se quejaban de insatisfacción sexual, mejoraron post cirugía. 
Tabla 1

COLPOPEXIA SACROSPINOSA

\begin{tabular}{|c|c|c|c|c|c|c|c|c|c|c|c|c|c|c|c|}
\hline Paciente \# & 01 & 02 & 03 & 04 & 05 & 06 & 07 & 08 & 09 & 10 & 11 & 12 & 13 & 14 & 15 \\
\hline Edad & 53 & 59 & 64 & 57 & 56 & 58 & 63 & 66 & 39 & 51 & 61 & 59 & 70 & 74 & 73 \\
\hline Fijación Exitosa & si & no & si & si & si & no & si & si & si & si & si & si*** & no & si & si \\
\hline Recidiva & no & $\mathrm{si}$ & no & no & no & si & no & no & no & no & no & $\mathrm{si}^{* * *}$ & si & no & no \\
\hline Sangrado & no & no & no & no & no & no & no & no & no & no & no & no & no & no & no \\
\hline $\begin{array}{l}\text { Infección } \\
\text { Dolor post }\end{array}$ & no & no & no & no & $* *$ si & no & no & no & no & no & no & no & no & no & no \\
\hline $\begin{array}{l}\text { Quirúrgico } \\
\text { Retención }\end{array}$ & no & no & no & no & no & no & no & no & no & no & no & $\mathrm{si}^{* * *}$ & no & no & no \\
\hline $\begin{array}{l}\text { Urinaria } \\
\text { Incontinencia }\end{array}$ & no & no & *no & no & no & no & no & no & no & no & no & no & no & no & no \\
\hline Urinaria & no & Leve & *no & no & no & no & no & no & no & no & no & no & no & no & no \\
\hline $\begin{array}{l}\text { Cistocele } \\
\text { Vida sexual pre- }\end{array}$ & G II & G III & G II & G II & G III & G III & G III & no & G II & G II & no & G III & G II & G III & G II \\
\hline $\begin{array}{l}\text { quirúrgica } \\
\text { Vida sexual post- }\end{array}$ & si & si & no & si & si & si & no & no & si & si & si & si & no & si & no \\
\hline $\begin{array}{l}\text { quirúrgica } \\
\text { Estancia }\end{array}$ & si & no & no & si & no & no & no & no & si & si & no & si & no & si & no \\
\hline $\begin{array}{l}\text { Hospitalaria } \\
\text { Aceptación }\end{array}$ & $\begin{array}{l}48 \mathrm{~h} \\
\mathrm{si}\end{array}$ & $\begin{array}{l}72 \mathrm{~h} \\
\text { no }\end{array}$ & $\begin{array}{l}72 \mathrm{~h} \\
*_{\mathrm{si}}\end{array}$ & $\begin{array}{l}48 \mathrm{~h} \\
\mathrm{si}\end{array}$ & $\begin{array}{l}* * 8 \mathrm{~d} \\
\mathrm{si}\end{array}$ & $\begin{array}{l}72 \mathrm{~h} \\
\text { no }\end{array}$ & $\begin{array}{l}72 \mathrm{~h} \\
\text { si }\end{array}$ & $\begin{array}{l}48 \mathrm{~h} \\
\mathrm{si}\end{array}$ & $\begin{array}{l}48 \mathrm{~h} \\
\mathrm{si}\end{array}$ & $\begin{array}{l}48 \mathrm{~h} \\
\text { si }\end{array}$ & $\begin{array}{l}60 \mathrm{~h} \\
\mathrm{si}\end{array}$ & $\begin{array}{l}72 \mathrm{~h} \\
\text { si }\end{array}$ & $\begin{array}{l}72 \mathrm{~h} \\
\mathrm{si}\end{array}$ & $\begin{array}{l}48 \mathrm{~h} \\
\text { si }\end{array}$ & $\begin{array}{l}72 \mathrm{~h} \\
\mathrm{si}\end{array}$ \\
\hline
\end{tabular}

* Sensación de masa vaginal al caminar, urgencia urinaria ocasional, se le "detiene" la orina al sentarse.

** Infección severa de cúpula post quirúrgica, hospitalización 8 días, no recidiva.

*** Se soltó el punto de fijación de la cúpula en el post quirúrgico inmediato, reintervenida con éxito.

\section{Estancia hospitalaria :}

El promedio de estancia fue de 72 horas con deambulación a las 24 horas y retiro de la sonda vesical en 24 a 48 horas (Exceptuando la paciente complicada con infección).

\section{Aceptación:}

Todas las 12 (doce) pacientes con cúpula sostenida manifestaron satisfacción con el procedimiento.

Una de las tres que presentaron recidiva insistió en que sentía gran mejoría, a pesar del evidente prolapso y rehusó otra cirugía.

Las dos restantes con cúpula prolapsada, acusaron sensación de masa en vagina y solicitaron ser reintervenidas.

\section{Resumen de resultados:}

Resultados exitosos 12 de 15

Complicaciones 01 de 15

Cistocele post Colpopexia 13 de 15

Estancia Hospitalaria 72 horas

\section{Análisis y conclusiones}

Si bien el número de resultados exitosos no alcanza al de otras publicaciones $(21-22,24)$, nuestra experiencia en un grupo no seleccionado de pacientes es aceptable, considerando que tres de las cuatro con recidiva del prolapso tenían cirugía previa por vía abdominal para correción del problema y habían fallado, siendo dos de ellas diabéticas.

En nuestras pacientes la presencia de cistocele residual llama la atención por su frecuencia y amerita una revisión de la técnica quirúrgica, aunque no se presentó incontinencia urinaria ni molestias relacionadas.

\section{Se concluye:}

La Colpopexia Sacrospinosa es una opción quirúrgica razonable para el manejo del Prolapso de Cúpula Vaginal, con una baja tasa de complicaciones, corta estancia hospitalaria, que puede ser empleada en pacientes con vida sexual activa, que presenten prolapso de cúpula con cisto-recto-enterocele y que amerita estudios más amplios comparativos con las técnicas abdominales para establecer indicaciones por grupos de edad, antecedentes y características físicas.

\section{Agradecimientos}

A los doctores Oscar Gómez Ceballos y Orlando Osorio, quienes introdujeron la técnica quirúrgica motivo de esta revisión en el armamentario de los ginecólogos a nivel nacional. Al doctor Germán Eugenio Osorio quien contribuyó a la realización de este estudio y practica la colpopexia con éxito. A los doctores Marco Alfonso Nieto y Juan Farid Sánchez por su colaboración en el análisis y redacción del artículo. 


\section{BIBLIOGRAFIA}

1. Drutz Harold P., Cha, Lilis S. Massive genital and vaginal vault prolapse treated by abdominal - vaginal sacropexy with use of Marlex mesh : Review of the literature. Am. J. Obstet. Gynecol. 1987; 156(2) : 387-392.

2. Baker, Kevin A., Beresford, James M, Campbell, Craig. Colposacropexy with Prolene mesh. Surg. Gynecol. Obstet. 1990; 171: 51-54.

3. Angulo A ., Kligman I. Retroperitoneal sacrocolpopexy for correction of the prolapse of the vaginal vault. Sur. Gynecol. Obstet. 1989; 169: 319-323.

4. Creighton, Sarah M., Stanton, Sturt L. The surgical management of the vaginal vault prolapse. Br. J. Obstet. Gynaecol. 1991; 98: 11501155 .

5. Rust, Jesse A., Bottle Joseph M., Howlett Ralph J. Prolapse of the vaginal vault. Am. J. Obstet. Gynecol. 1976; 125(6): 768-776.

6. Addison W., Allen Cols. Abdominal sacral colpopexy with Mersilene mesh in the retroperitoneal position in the management of the post hysterectomy vaginal vault prolapse and enterocele. Obstet. Gynecol. 1985 ; 153(2): 140-146.

7. Gómez PI. Tratamiento del prolapso de cúpula vaginal con sacrocolpopexia modificada utilizando dacrón de injerto vascular. Rev. Col. Ginecol. 1995; 46( 3): 197-200.

8. Virtanen H., Hirvonen T., Makinen J., Kilholma P. Outcome of thirty patients who underwent repair of posthysterectomy prolapse of the vaginal vault with abdominal sacral colpopexy. J. Am. Coll. Surg. 1994; 178(3): 283-287.

9. Given FT., Jr, Muhlendorf, IK., Browning GM. Vaginal and sexual function after colpopexy for complete uterovaginal eversion. Am. J. Obstet. Gynecol. 1993; 169 (2 pt 1): 284-287.

10. Nichols, David H. Sacrospinous fixation for the massive eversion of the vagina. Am. J. Obstet. Gynecol. 1982; 142: 901-904.

11. Nichols DH. Surgery for pelvic floor disorders. Surg. Clin. N. A . 1991; 75(5): 927- 946.

12. Brieger GM., MacGibbon AL., Atkinson KH. Sacrospinous Colpopexy. Aust N Z J Obstet. Gynecol. 1995; 35(1): 86- 87.

13. Zweifel P. Vorlesungen über Klinische Gynäkologie. Berlin; Hirschwald: $1892 ; 407$.
14. Miller NF. A new method of correcting complete inversion of the vagina. Surg. Gynecol. Obstet. 1927; 44: 550.

15. Mengerts WF. Am. J. Obstet. Gynecol. 1936; 31: 775.

16. Amreich J. Aetiologie und operation des scheidenstumpf prolapses .Wien Klin Wschr 1951; 63: 74-77.

17. McCall M L. Posterior culdeplasty: Surgical correction of enterocele during vaginal hysterectomy, a preliminar report. Obstet. Gynecol. 1957; 10: 595-602.

18. Richter K. Die operative behandlung des prolabierten scheiden grundes nach utersextirpation. Ein beitrag zur vaginaefixatio sacrotuberalis nach Amreich. Geburts Frauenheilk 1967; 27: 941.

19. Richter K. Lebendige anatomic der vagina. Geburts u Frauenh 1966; 26: 1213 .

20. Richter K. Die chirurgische anatomic der vaginaefixatio sacrospinalis vaginalis. Ein bietrag zur operativen behandlung des scheidenblindsackprolapses. Geburts u Frauen 1968; 28: 321-327.

21. Randall C L., Nichols DH. Surgical treatment of vaginal inversion. Obstet. Gynecol. 1971; 38(3): 327-332.

22. Morley GW.,DeLancey OL. Sacrospinous ligament fixation for eversion of the vagina . Am. J. Obstet. Gynecol. 1988; 158(4): 872-881.

23. Peters W A., Christenson ML . Fixation of the vaginal apex to the coccygeus fascia during repair of the vaginal vault eversion with enterocele. Am. J. Obstet. Gynecol. 1995; 172(6): 1894-1900.

24. Richter K. Massive eversion of the vagina: Pathogenesis, diagnosis, and therapy of the "true" prolapse of the vaginal stump. Clin. Obstet. Gynecol. 1982; 25(4): 897-911.

25. Kettel L., Michael, Hebertson, Richard M. An anatomic evaluation of the sacrospinous ligament fixation. Surg. Gynec. Obstet. 1989; 168: 318-322.

26. Nichols David H., Milley Raul S., Randall Clyde L . Significance of restoration of normal vaginal depth an axis.Obstet. Gynecol. 1970; 36(2): 251-255.

27. Morley George W. Tratamiento para la eversión de la cúpula vaginal a través de la vía vaginal. Clin. Obstet. Gynecol. 1993 ; 4: 925-933.

28. Farrel Scott A., Bent, Alfred E. Massive evisceration: A complication following sacrospinous vaginal vault fixation. Obstet. Gynecol. 1991; 78: $560-562$. 